\title{
Application of artificial neural network for control and navigation of humanoid robot
}

\author{
Asita Kumar Rath ${ }^{1 *}$, Harish Chandra Das ${ }^{2}$, Dayal R. Parhi ${ }^{3}$, \\ Priyadarshi Biplab Kumar ${ }^{4}$
}

\author{
${ }^{1}$ Centre of Biomechanical Science, Siksha ' $O$ ' Anusandhan Deemed to be University, \\ Bhubaneswar, Odisha, India-751030 \\ Email: asitr06@gmail.com ${ }^{1 *}$ \\ Phone: +91-9040581281 \\ ${ }^{2}$ Mechanical Engineering Department, National Institute of Technology, Shillong, \\ Meghalaya, India-793003 \\ ${ }^{3}$ Robotics Laboratory, Mechanical Engineering Department, National Institute of \\ Technology, Rourkela, Odisha, India-769008 \\ ${ }^{4}$ Robotics Laboratory, Mechanical Engineering Department, National Institute of \\ Technology, Rourkela, Odisha, India-769008
}

\begin{abstract}
With the development of science and technology, humanoid robots are widely used among several industries. Humanoid robots are seen as a human replacement in a vast sense. It is a test for analysts to imitate the human aptitude in a counterfeit humanoid robot movement framework. With the developing innovation, the humanoid robots are being created for planetary investigation alongside other versatile robots to additionally enhance the mobility in a thickened domain. This paper is focussed on the development of an Artificial Neural Network based navigational controller for path planning examination of humanoid robot strolling. The path planning analysis is carried out on a NAO humanoid robot. Sensory information regarding obstacle distances and location of target are fed as inputs to the controller and required streaming angle is obtained as the output. Navigational analysis has been performed in both simulation and experimental environments with complicated arena conditions. Finally, a comparison between simulation and experimental results has been done, and the result are found to be in good agreement.
\end{abstract}

Keywords: Humanoid Robot; Navigation; Path planning; ANN.

\section{INTRODUCTION}

Robotics have been the centre of attraction for many researchers over the last few decades. Ito et al. [1] have studied about different types of ball handling behaviours of a small humanoid robot in a dynamic neural network model. Patacchiola et al. [2] have proposed a neural network approach for head pose estimation. They evaluated four architecture performances for the wild data sets. Pothal et al. [3-5] used a neural network approach combined with fuzzy logic system for navigation of multiple mobile robots in highly cluttered environments. Savage et al. [6] have generated the behaviours of a mobile robot by using Genetic Algorithm (GA). Bajrami et al. [7] have used both fuzzy logic controller (FLC) and artificial neuronal fuzzy logic (ANFL) for avoiding both static and dynamic obstacles in a mobile robot platform. Parhi et al. [8-10] used different artificial intelligent 
(AI) techniques for path optimization of robotic agents and validated their approach through multiple simulations and experiments. Park et al. [11] have studied the "communicable congruence" with human in an experimental neuro-robotics. They controlled humanoid robots by the neuromorphic model with the utilization of the dynamic character in a neural network model. Pradhan et al. [12-15] used fuzzy logic as a potential navigational algorithm for both path and time optimization of a robotic agent to move from a definite initial position to a desired target location. Son et al. [16] combined feed forward neural network controller with a PID module to control a SCARA parallel robot joint angle position. Tamayo et al. [17] have developed and analysed the AH1N2 humanoid robot. Mohanta et al. [18-19] applied genetic algorithm for systematic search of a safe path in the navigation of a mobile robot towards its target location. Sun et al. [20] have discussed about the direction following control of an adaptable connection space controller. They have used and analysed different AI technique for solving various problem related to robot navigation. Several researchers [21-24] have also tried to modify basic AI algorithms for performance improvement in engineering problems. Virgala et al. [25] have supervised examining, demonstrating and recreation of movement of humanoid robot hand with 24 degrees of freedom (DOF). Deepak et al. [26-29] used artificial immune based and other related approach in mobile robot navigation. Mohanty et al. [3033] developed different nature inspired algorithms for smooth planning of mobile robot navigation. Elliot et al. [34] have conducted several experiments for the accomplishment of a new control architecture design for the mobile robots which help in the autonomy of disabled people. Singh et al. [35-38] used neural network as a potential navigation approach for multiple robotic agents. Singh and Nandi [39] analysed different calibration techniques for a humanoid in drawing different objects. Kundu et al. [40-43] used different AI techniques for navigation of underwater mobile robots. They considered different constraints that may arise while working with a robot in underwater conditions. Madani et al. [44] have proposed a machine-learning based multi-level mental model induced from early-ages' academic movement of human's advancement capacities with regards to humanoid robot's strolling appearing. Nuovo et al. [45] discussed regarding a memory imagination based analysis for a humanoid robot.

From the extensive survey of the available literature, it can be conceived that navigation and path planning have been mostly applied to mobile robots only. A very few works have been reported towards humanoid navigation. Therefore, the current work is devoted towards the design and development of an artificial neural network (ANN) based control architecture for navigation of a humanoid robot in a complex environment. Here, NAO has been chosen as the humanoid platform for navigational analysis. The performance of the controller has been verified through multiple simulations and experiments. Finally, the results obtained from both the environments are compared against each other with good agreement between them.

\section{ARTIFICIAL NEURAL NETWORK}

ANN system is inspired from the human brain, and its functional architecture is similar to biological neural systems. The characteristics of the human brain like complex and non-linear information processing in parallel time have inspired the development of an artificial neural system to solve different engineering problems. To design an artificial neural system, neuron modelling, layer designing, and learning algorithm are important which have been briefly described as follows. 


\section{Neuron Modelling}

A biological neuron consists of a cell body connected to dendrites and axons that receives and conveys the signal from one cell body to another respectively. The biological neurons comprise of body cells, dendrites, axons, and synapses. The information exchange takes place in the form of electrical signals between the neurons through dendrites and axons at the junction space called synapse. McCulloch and Pitt recreated the biological neurons characteristics in the form of neuron model.

\section{Architecture}

The architecture of ANN that connects neurons to other neurons forms a net which is distinguished into three layers. The first one is an input layer that receives the signal from another stimulated neuron. The data is forwarded to the next layer which consists of one or more neurons that can be chosen independently also known as an unseen layer that processes the data with suitable mathematical operations. The processed data is then transferred to the output layer that analyzes the data by the use of activation function The architecture of the ANN system can be feed forward or feedback type in nature. The feed forward does not send back the signal to the previous layer, and the data is allowed to propagate in a forward direction towards the output. On the other hand, the feedback ANN the data could propagate backward, and such a system is used in dynamic applications. Figure 1 represents the basic control architecture of an ANN model.

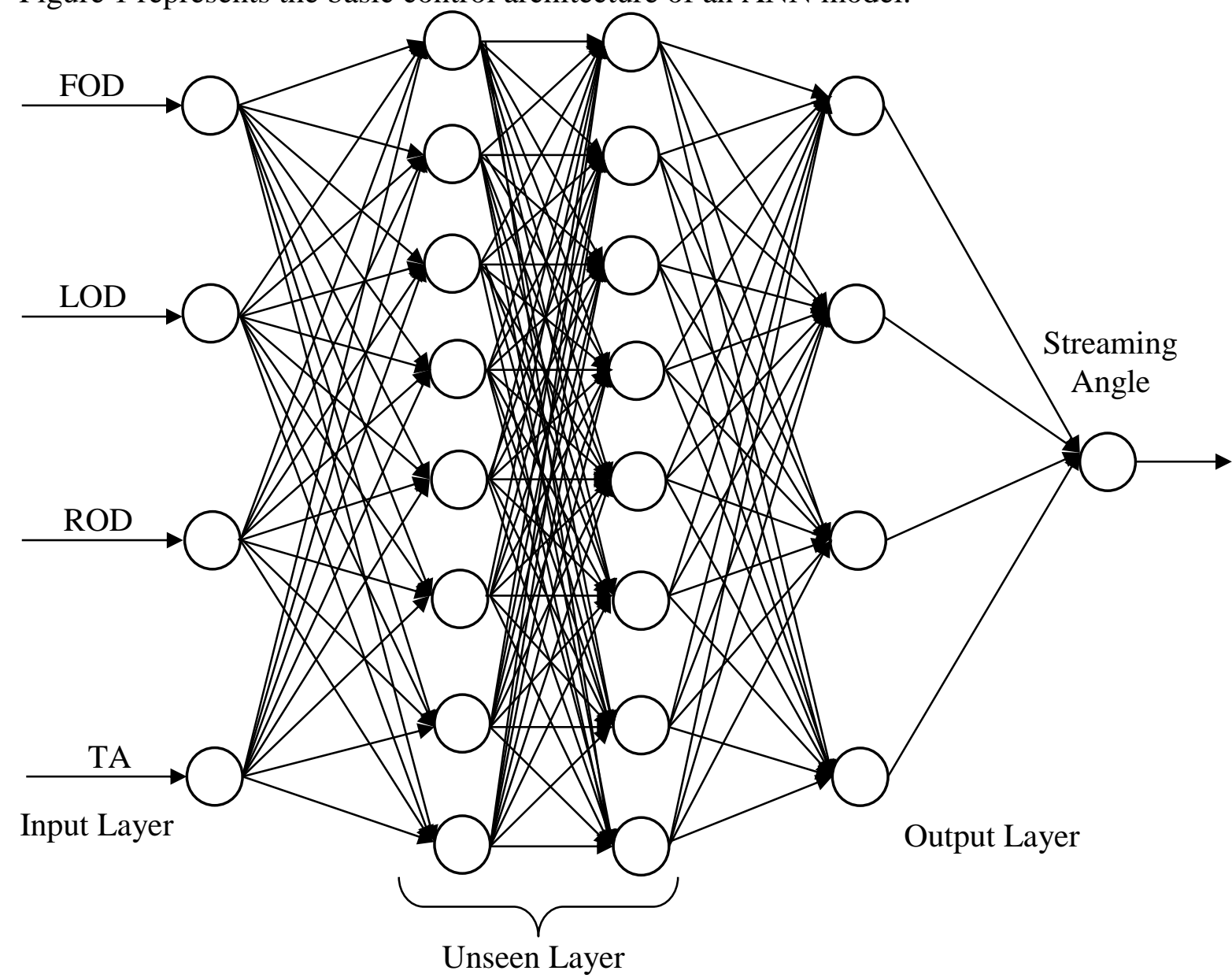

Figure 1. ANN architecture. 
The input value fed to the input layer, and the result distributed to the unseen layer and turn the neural network generate output is given by:

Where,

$$
Y_{j}^{(l a y)}=f\left(V_{j}^{(l a y)}\right)
$$

(lay) $=$ Unseen layer

$j=j^{\text {th }}$ neuron in unseen layer "lay"

$i=i^{\text {th }}$ neuron in unseen layer "lay-1"

$W_{j i}^{(l a y)}=$ weight of the neuron.

For the neural network controller, the input parameters are as follows.

Front obstacle distance $=$ FOD

Left obstacle distance $=$ LOD

Right obstacle distance $=$ ROD

Target angle $=$ TA

The controller output is steering angle.

By using the MATLAB programming, the ANN has been written, and all the tests are done in a personal computer. For this work, the hyperbolic tangent function is chosen as activation function.

$$
\mathrm{F}(\mathrm{x})=\frac{\mathrm{a}^{\mathrm{x}}-\mathrm{a}^{-\mathrm{x}}}{a^{x}+a^{-x}}
$$

The output of the network $\varphi_{\text {actual }}$ unlike from the $\varphi_{\text {disered }}$ in the training pattern presented to the network. The performance of the network is the sum-squared difference between $\varphi_{\text {actual }}$ and $\varphi_{\text {disered. }}$

$$
\text { Error }=\frac{1}{2} \sum(\varphi \text { disered }-\varphi \text { actual })^{2}
$$

For calculating the error in the network back propagation method used. This method is used to measure the computational error gradient to find out the exact weight correction to minimise the error of the output layer.

The error gradient is as follows.

$$
\lambda^{(4)}=f^{\prime}\left(V_{1}^{(4)}\right)\left(\varphi_{\text {desired }}-\varphi_{\text {actual }}\right)
$$

For the unseen layer, the local gradient for neuron is given by:

$$
\varphi_{f}^{(\text {lay })}=f^{\prime}\left(V_{j}^{(\text {lay })}\right)\left(\sum_{k} \lambda_{k}^{(\text {lay })} W_{i j}^{(l a y+1)}\right)
$$

The weight is given as:

$$
W_{j i}(n+1)=W_{j i}(n)+\Delta W_{j i}(n+1)
$$

And change in weight is given as:

$$
\Delta W_{j i}(n+1)=\beta \Delta W_{j i}(n)+\alpha \lambda_{j}^{(\text {lay })} Y_{i}^{(l a y-1)}
$$

Where $\beta=$ The momentum coefficient $\cong 0.3, \alpha=$ Learning rate $\cong 0.35, n=$ Iteration number.

\section{Learning Algorithm}

Sometimes to attain accuracy for a given system, the input parameters and the weight of synapses need to be modified, and ANN's learning algorithm gives the steady reaction to inputs as desired. ANN Learning is carried out to produce the desired output by regulating the input parameters. Some authors have suggested three necessary steps to be followed for designing ANN learning algorithm. The learning model is the first step that describes the process of learning in accordance with the system. The learning algorithm is the next 
step that refers to the rules adapted to modify the parameters and synapses weight. The final step is the boundary condition which puts the limitation on the learning ability. It refers to the capacity of learning, samples and time requirement for training the ANN. The learning process is of two types supervised and unsupervised that have been widely used. The information received by the supervised learning is in the quotation form whereas the data that an unsupervised learning receives is random.

\section{IMPLEMENTATION OF ANN MODEL IN HUMANOID NAVIGATION}

The path planning for humanoid robot in simulation environment has been carried out using V-REP software with one NAO robot in the scene and five numbers of obstacles. Figure 2 and Figure 4 show the simulated path obtained by application of ANN in the humanoid platform. To compare the simulation results, an experimental platform was also prepared under laboratory conditions. Figure 3 and Figure 5 show the experimental path obtained by application of ANN. The results obtained from both the platforms are compared against each other. Table 1 determines the error in path length and Table 2 shows the error in the time taken between simulation and experimental platform using the ANN approach.

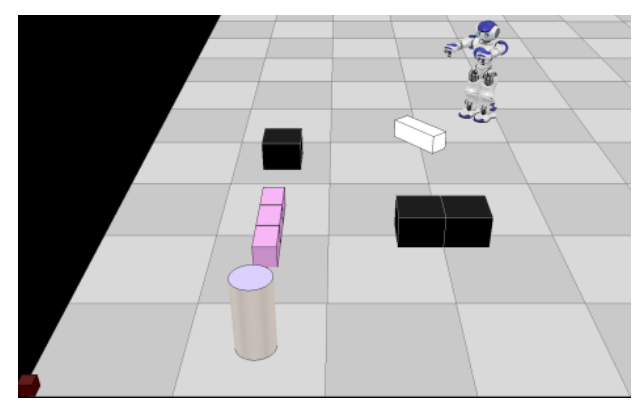

(a)

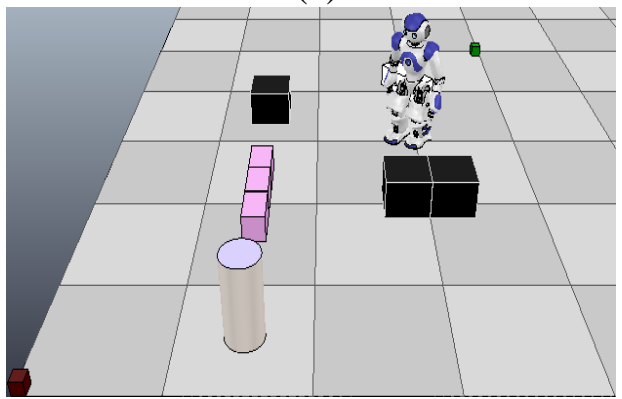

(c)

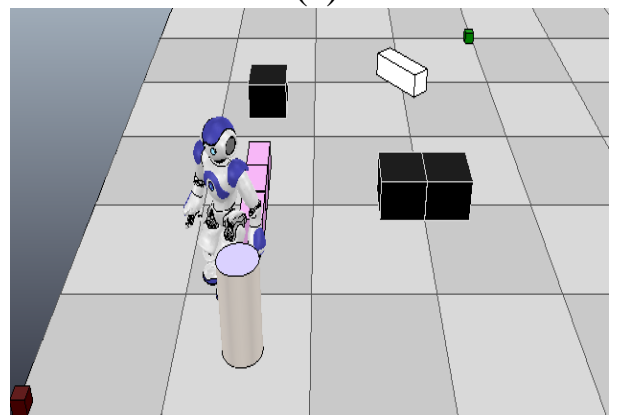

(e)

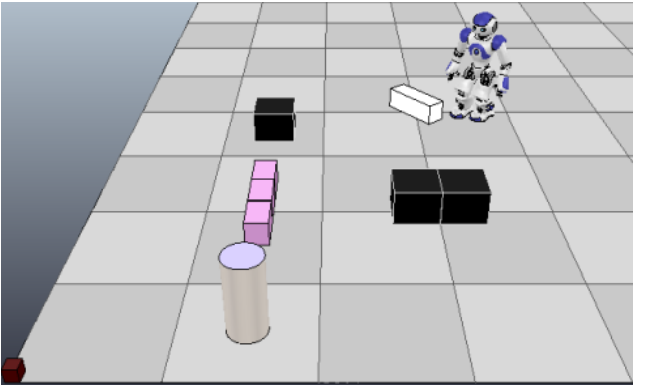

(b)

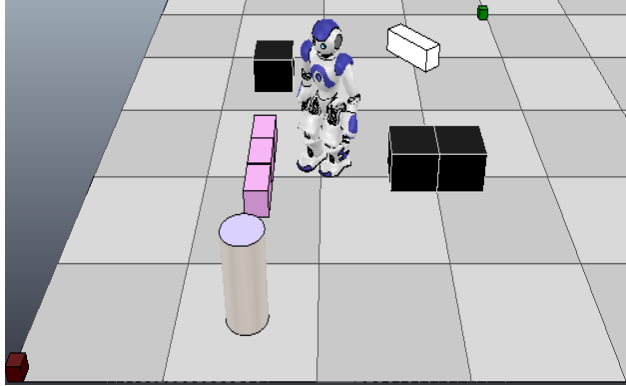

(d)

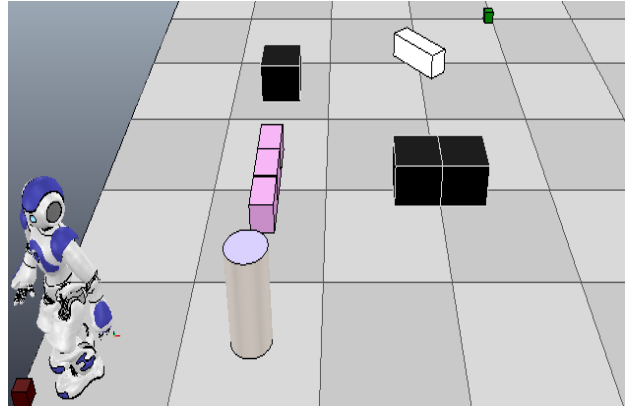

(f)

Figure 2. Simulation results for scene 1. 


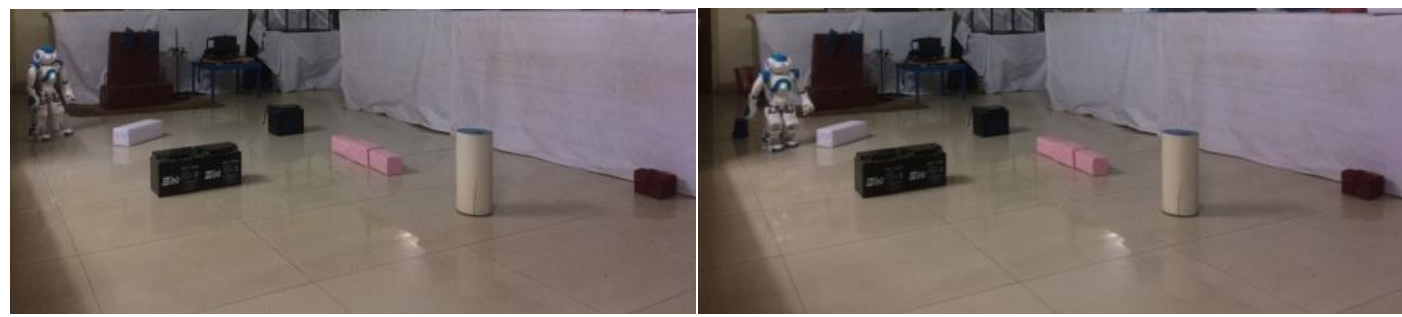

(a)

(b)

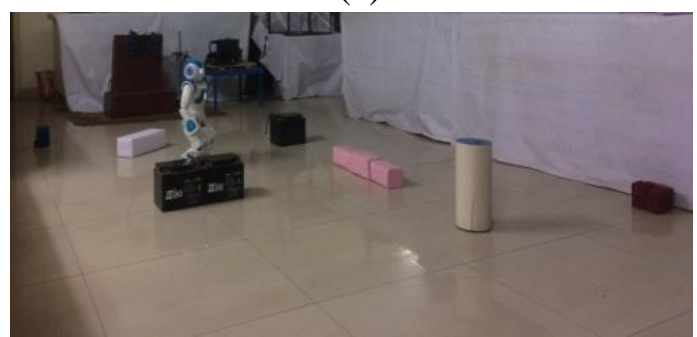

(c)
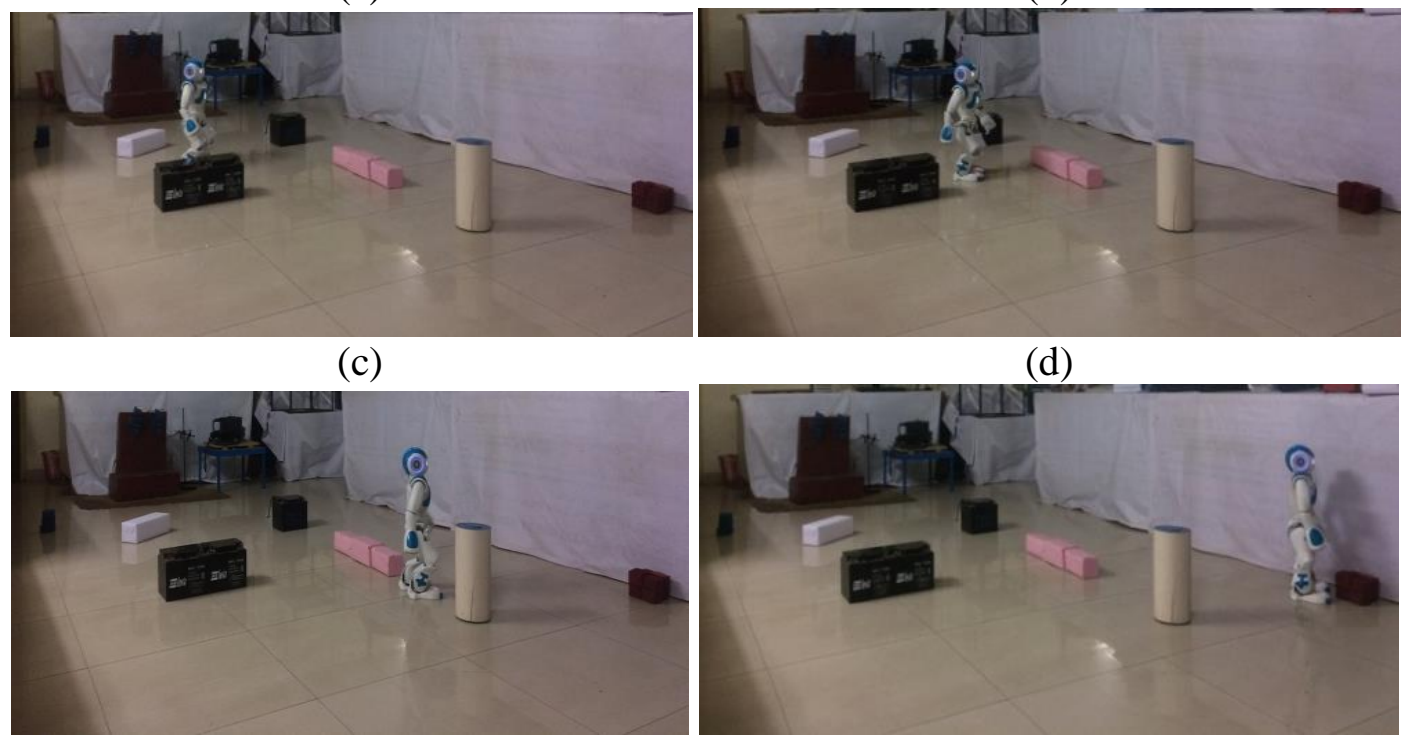

(d)

(e)

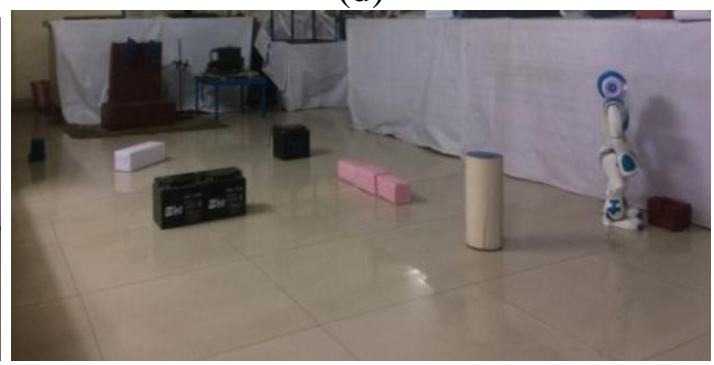

(f)

Figure 3. Experimental results for scene 1.

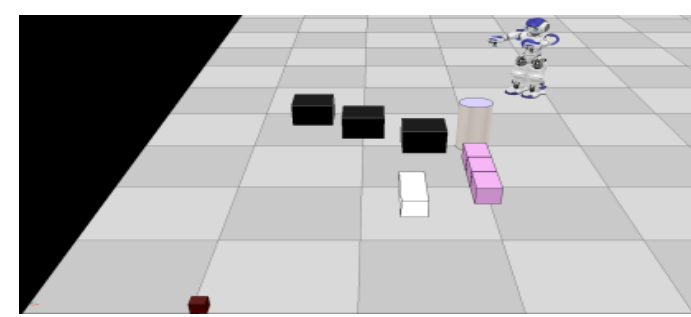

(a)

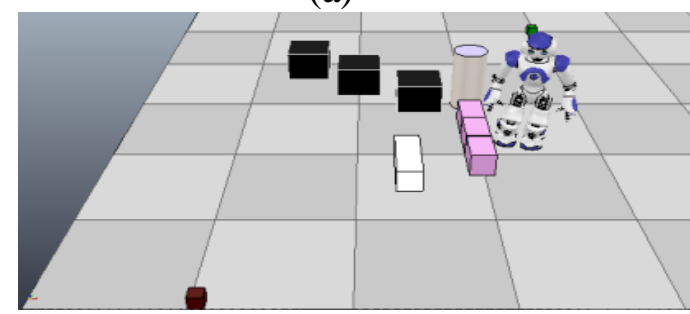

(c)

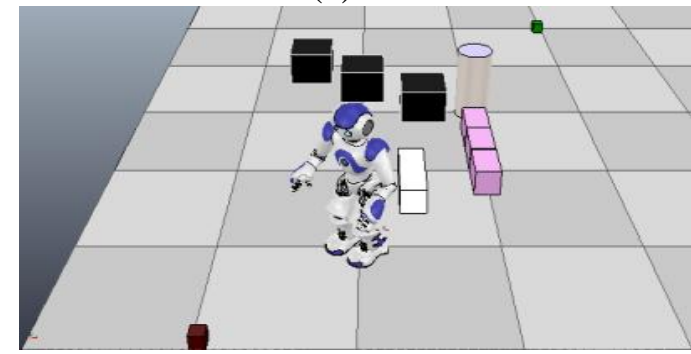

(e)

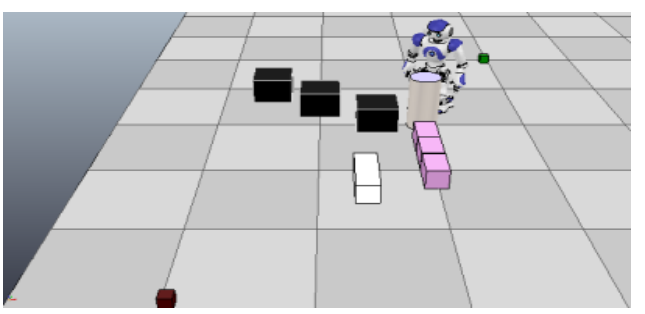

(b)

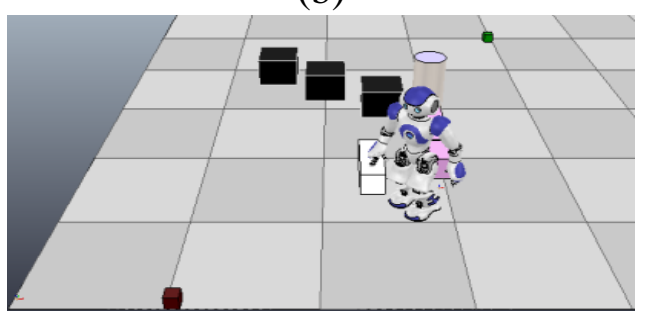

(d)

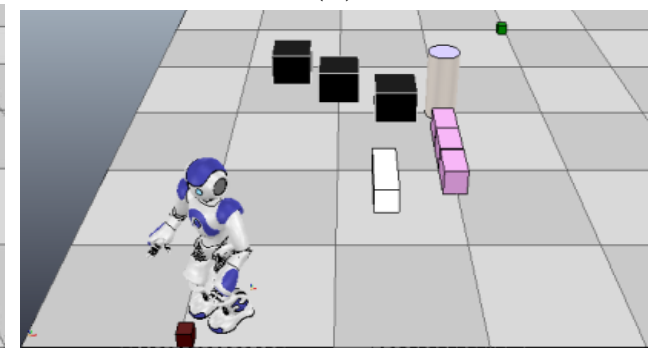

(f)

Figure 4. Simulation results for scene 2. 


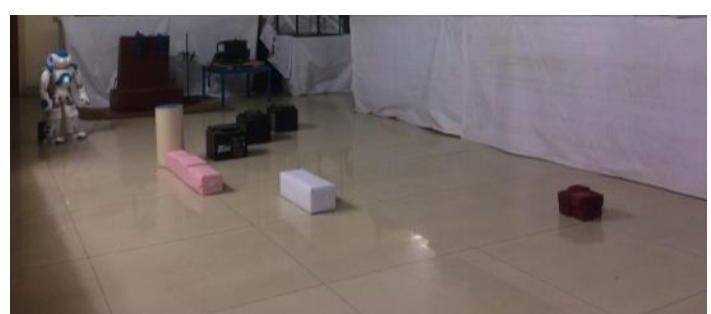

(a)

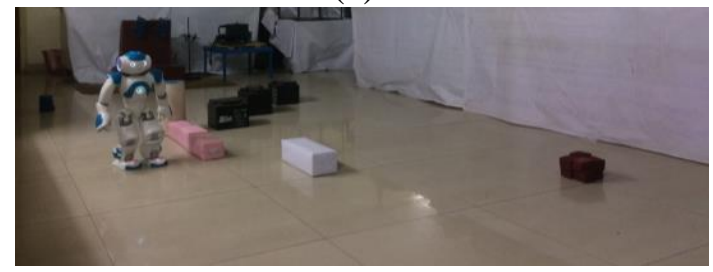

(c)

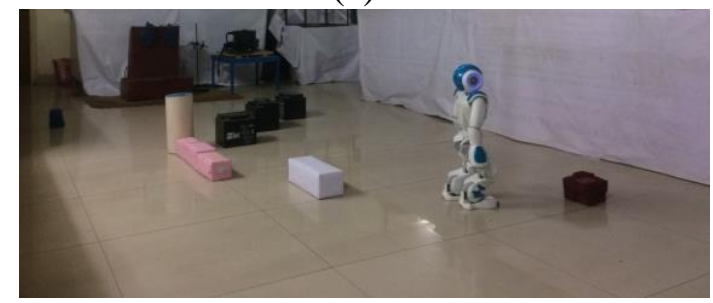

(e)

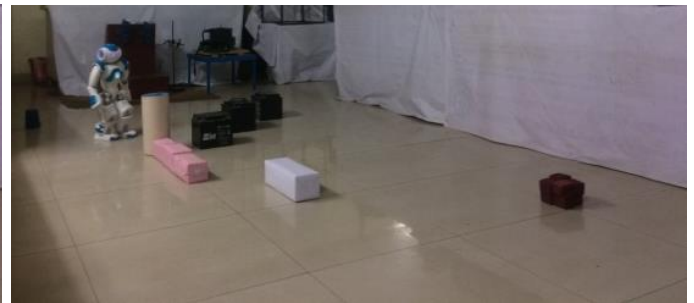

(b)

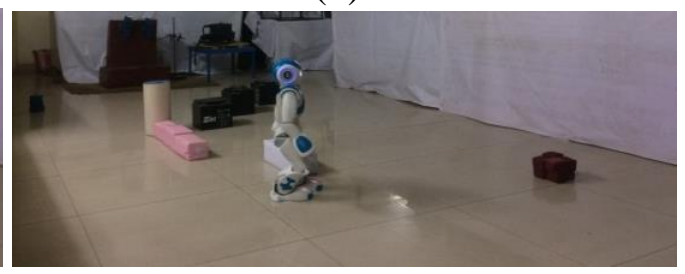

(d)

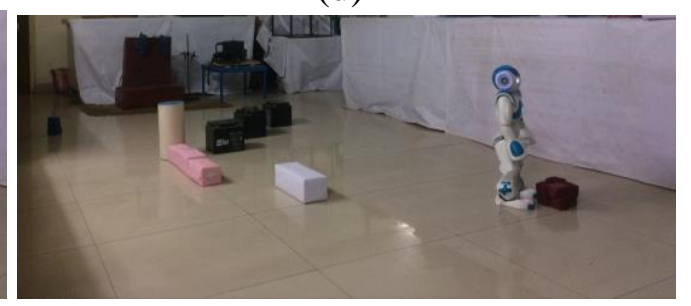

(f)

Figure 5. Experimental results for scene 2.

Table 1. Comparison of simulated and experimental path length.

\begin{tabular}{cccc}
\hline Sl. No. & $\begin{array}{c}\text { Simulation path } \\
\text { length }(\mathrm{m})\end{array}$ & $\begin{array}{c}\text { Experimental path } \\
\text { length }(\mathrm{m})\end{array}$ & $\begin{array}{c}\text { Error in path } \\
\text { length }(\%)\end{array}$ \\
\hline 1 & 3.18 & 3.41 & 6.74 \\
2 & 3.08 & 3.36 & 8.33 \\
3 & 3.05 & 3.25 & 6.15 \\
4 & 3.2 & 3.4 & 5.8 \\
5 & 3.01 & 3.15 & 4.44 \\
\hline
\end{tabular}

Table 2. Comparison of simulated and experimental time taken.

\begin{tabular}{cccc}
\hline Sl. No. & $\begin{array}{c}\text { Simulation } \\
\text { Time taken(s) }\end{array}$ & $\begin{array}{c}\text { Experimental time } \\
\text { taken }(\mathrm{s})\end{array}$ & $\begin{array}{c}\text { Error in time } \\
(\%)\end{array}$ \\
\hline 1 & 55.5 & 57.4 & 3.31 \\
2 & 53.0 & 55.9 & 5.18 \\
3 & 49.9 & 52.1 & 4.22 \\
4 & 54.5 & 57.5 & 5.21 \\
5 & 50.7 & 53.3 & 4.87 \\
\hline
\end{tabular}

The path obtained in every scene using ANN gives an optimal solution to the problem; so ANN method can be used as a potential navigational controller that can provide the least path length and time taken from start point to end point. It can be noticed that the experimental results always show higher values than the simulation ones. The simulation platform is an ideal one where there is absence of any loss factor. However, there are presence of different factors like slippage of robot's foot, frictional losses, loss in data transmission, etc. in the experimental platform which cause the higher values. 


\section{CONCLUSION}

Path planning for humanoid robot requires sensory system and the conditions in local environment. The classical methods applied to avoid obstacles and reach the target don't provide the optimized path that a robot could manoeuvre since intelligent approaches could provide an efficient path for the robot to reach the target following a least path in the minimum possible time. Therefore, the current paper is dedicated to obtain such a path using ANN method in a cluttered environment. Path planning using ANN method proves to be more efficient. Therefore, ANN can be successfully used for optimized humanoid robot path planning in a cluttered environment.

\section{REFERRENCE}

[1] Ito M, Noda K, Hoshino Y, Tani J. Dynamic and interactive generation of object handling behaviors by a small humanoid robot using a dynamic neural network model. Neural Networks. 2006;19(3):323-337.

[2] Patacchiola M, Cangelosi A. Head Pose Estimation in the Wild using Convolutional Neural Networks and Adaptive Gradient Methods. Pattern Recognition, 2017.

[3] Pothal JK, Parhi DR. Navigation of multiple mobile robots in a highly clutter terrains using adaptive neuro-fuzzy inference system. Robotics and Autonomous Systems, 2015; 72:48-58.

[4] Singh MK, Parhi DR, Pothal JK. ANFIS approach for navigation of mobile robots. In: IEEE International Conference on Advances in Recent Technologies in Communication and Computing. 2009;727-731.

[5] Parhi DR, Pothal JK, Singh MK. Navigation of multiple mobile robots using swarm intelligence. In: IEEE World Congress on Nature \& Biologically Inspired Computing. 2009; 1145-1149.

[6] Savage J, Muñoz S, Matamoros M, Osorio R. Obstacle Avoidance Behaviors for Mobile Robots Using Genetic Algorithms and Recurrent Neural Networks. IFAC Proceedings. 2013;46(24):141-146.

[7] Bajrami X, Dërmaku A, Demaku, N. Artificial neural fuzzy logic algorithm for robot path finding. IFAC-Papers OnLine. 2015;48(24):123-127.

[8] Parhi DR, Pothal JK. Intelligent navigation of multiple mobile robotsusing an ant colony optimization techniquein a highly cluttered environment. Proceedings of the Institution of Mechanical Engineers, Part C: Journal of Mechanical Engineering Science. 2011;225(1):225-32.

[9] Parhi DR, Inala R, Pothal JK. Cooperative Behavior of Multiple Mobile Robots by Using Swarm Intelligence Technique.

[10] Pham DT, Parhi DR. Navigation of multiple mobile robots using a neural network and a Petri Net model. Robotica. 2003;21(1):79-93.

[11] Park G, Tani J. Development of compositional and contextual communicable congruence in robots by using dynamic neural network models. Neural Networks. 2015;72:109-122.

[12] Pradhan SK, Parhi DR, Panda AK. Fuzzy logic techniques for navigation of several mobile robots. Applied soft computing. 2009;9(1):290-304.

[13] Pradhan SK, Parhi DR, Panda AK. Neuro-fuzzy technique for navigation of multiple mobile robots. Fuzzy Optimization and Decision Making. 2006;5(3):255-88. 
[14] Pradhan SK, Parhi DR, Panda AK, Behera RK. Potential field method to navigate several mobile robots. Applied Intelligence. 2006;25(3):321-33.

[15] Pradhan SK, Parhi DR, Panda AK. Navigation of multiple mobile robots using rule-based neuro-fuzzy technique. International Journal of Computational Intelligence. 2006;3(2):142-52.

[16] Son NN, Van Kien C, Anh HPH. A novel adaptive feed-forward-PID controller of a SCARA parallel robot using pneumatic artificial muscle actuator based on neural network and modified differential evolution algorithm. Robotics and Autonomous Systems. 2017;96:65-80.

[17] Tamayo AJM, Bustamante PV, Ramos JJM, Cobo AE. Inverse models and robust parametric-step neuro-control of a Humanoid Robot. Neurocomputing. 2017;233:90-103.

[18] Parhi DR, Mohanta JC. Navigational control of several mobile robotic agents using Petri-potential-fuzzy hybrid controller. Applied Soft Computing. 2011;11(4):3546-3557.

[19] Mohanta JC, Parhi DR, Patel SK. Path planning strategy for autonomous mobile robot navigation using Petri-GA optimisation. Computers \& Electrical Engineering. 2011;37(6):1058-70.

[20] Sun F, Zhang L, Sun Z. Neuro-fuzzy Inverse Dynamics Control for Flexible-link Space Robots. IFAC Proceedings. 2001;34(22):203-208.

[21] Deepak BBVL, Parhi DR, Kundu S. Innate immune based path planner of an autonomous mobile robot. Procedia Engineering. 2012;38:2663-2671.

[22] Deepak BBVL, Parhi DR. Kinematic analysis of wheeled mobile robot. Automation \& Systems Engineering. 2011;5(2).

[23] Deepak BB, Parhi DR, Raju BM. Advance particle swarm optimization-based navigational controller for mobile robot. Arabian Journal for Science and Engineering. 2014;39(8):6477-87.

[24] Deepak BB, Parhi D. Intelligent adaptive immune-based motion planner of a mobile robot in cluttered environment. Intelligent Service Robotics. 2013;6(3):155-62.

[25] Virgala I, Kelemen M, Varga M, Kuryło P. Analyzing, Modeling and Simulation of Humanoid Robot Hand Motion. Procedia Engineering. 2014;96:489-499.

[26] Deepak BB, Parhi DR. Control of an automated mobile manipulator using artificial immune system. Journal of Experimental \& Theoretical Artificial Intelligence. 2016;28(1-2):417-39.

[27] Deepak BB, Parhi D. PSO based path planner of an autonomous mobile robot. Open Computer Science. 2012;2(2):152-68.

[28] Deepak BB, Parhi DR. Target seeking behaviour of an intelligent mobile robot using advanced particle swarm optimization. In: IEEE International Conference on Control, Automation, Robotics and Embedded Systems (CARE). 2013;1-6.

[29] Deepak BB, Parhi DR, Jha AK. Kinematic Model of Wheeled Mobile Robots. Int. J. on Recent Trends in Engineering \& Technology. 2011;5(04).

[30] Mohanty PK, Parhi DR. Controlling the motion of an autonomous mobile robot using various techniques: a review. Journal of Advance Mechanical Engineering. 2013;1(1):24-39.

[31] Mohanty PK, Parhi DR. Optimal path planning for a mobile robot using cuckoo search algorithm. Journal of Experimental \& Theoretical Artificial Intelligence. 2016;28(1-2):35-52. 
[32] Mohanty PK, Parhi DR. A new intelligent motion planning for mobile robot navigation using multiple adaptive Neuro-fuzzy inference system. Applied Mathematics \& Information Sciences. 2014;8(5):2527.

[33] Mohanty PK, Parhi DR, Jha AK, Pandey A. Path planning of an autonomous mobile robot using adaptive network based fuzzy controller. In: IEEE 3rd International Advance Computing Conference (IACC). 2013;651-656.

[34] Eliot E, BBVL D, Parhi DR. Design \& kinematic analysis of an articulated robotic manipulator. 2012.

[35] Singh MK, Parhi DR. Path optimisation of a mobile robot using an artificial neural network controller. International Journal of Systems Science. 2011;42(1):107120.

[36] Parhi DR, Singh MK. Navigational strategies of mobile robots: a review. International Journal of Automation and Control. 2009;3(2-3):114-134.

[37] Singh MK, Parhi DR. Intelligent neuro-controller for navigation of mobile robot. In: Proceedings of the International conference on advances in computing, communication and control. 2009;123-128.

[38] Singh MK, Parhi DR, Bhowmik S, Kashyap SK. Intelligent controller for mobile robot: Fuzzy logic approach. In: The 12th International Conference of International Association for Computer Methods and Advances in Geomechanics (IACMAG). 2008;1-6.

[39] Singh AK, Nandi GC. NAO humanoid robot: Analysis of calibration techniques for robot sketch drawing. Robotics and Autonomous Systems. 2016;79:108-121.

[40] Kundu S, Parhi DR. Navigation of underwater robot based on dynamically adaptive harmony search algorithm. Memetic Computing. 2016;8(2):125-146.

[41] Kundu S, Parhi DR, Deepak BB. Fuzzy-neuro based navigational strategy for mobile robot. International Journal of Scientific \& Engineering Research. 2012;3(6):1-6.

[42] Kundu S, Parhi DR. Behavior-based navigation of multiple robotic agents using hybrid-fuzzy controller. In: IEEE International Conference on Computer and Communication Technology (ICCCT). 2010;706-711.

[43] Kundu S, Dayal RP. A fuzzy approach towards behavioral strategy for navigation of mobile agent. In: IEEE International Conference on Emerging Trends in Robotics and Communication Technologies (INTERACT). 2010;292-297.

[44] Madani K, Sabourin C. Multi-level cognitive machine-learning based concept for human-like "artificial" walking: application to autonomous stroll of humanoid robots. Neurocomputing. 2011;74(8):1213-1228.

[45] Di Nuovo AG, Marocco D, Di Nuovo S, Cangelosi A. Autonomous learning in humanoid robotics through mental imagery. Neural Networks. 2013;41:147-155. 